\title{
Frequency Stability in Modern Power Network from Complex Network Viewpoint
}

\author{
Hai-Peng Ren ${ }^{\mathrm{a},}$, Yuan Gao ${ }^{\mathrm{a},}$, Long Huo ${ }^{\mathrm{a}}$, Ji-hong Song ${ }^{\mathrm{a}}$, Celso Grebogi ${ }^{\mathrm{a}, \mathrm{b}}$, \\ ${ }^{a}$ Shaanxi Key Laboratory of Complex System Control and Intelligent Information Processing, \\ Xian University of Technology Xi'an, Shaanxi 710048, China \\ ${ }^{b}$ Institute for Complex Systems and Mathematical Biology, Kings College, University of \\ Aberdeen, Aberdeen AB24 3UE, UK
}

\begin{abstract}
Grid-connected operation of Renewable Energy and Storage (RES) nodes make the dynamics of modern power grid to be more complex. A model of power grid, considering RES nodes, is being proposed to address frequency synchronization and stability analysis. First, a unified dynamical model of four different types of nodes are established according to the swing equation, including the RES nodes with power-frequency droop inverter controllers. The storage nodes have charging and discharging states. We provide a sufficient condition for the existence and stability of frequency synchronized solution via the linear time-varying consensus protocol of multi-agent system. The results are validated by Western System Coordinating Council and the Shaanxi North Power Grid model.
\end{abstract}

Keywords:

renewable energy and storage, multi-agent system, frequency synchronization, stability, consensus protocol

\section{Introduction}

To solve the problem of energy consumption and carbon dioxide emission caused by the traditional fossil energy generation, the proportion of renewable energy generation is being increased in power grids. Grid-connected operation of intermittent energy, like solar energy or wind energy, leads to fluctuations in energy supplying and demanding, in spite of energy storage systems reduce such fluctuations. The modern power grid, therefore, is a time-varying, nonlinear coupled

Email address: renhaipeng@xaut . edu. cn (Hai-Peng Ren) 
network, heterogeneously composed of Renewable Energy and Storage (RES) nodes and the conventional generation nodes ${ }^{[1]}$. Therefore the traditional frequency synchronization methods are difficult to apply. The synchronization concept can be traced back to 1665 , when C. Huygens found that coupled pendula would synchronously swing. The Kuramoto model, proposed by biologist Winfree and Kuramoto, has made much contribution to the synchronization research. A review [2] summarized a series of research results about the Kuramoto oscillators, including the stability analysis. Kuramoto model with a bimodal parameter distribution was proposed to describe the synchronous oscillation of the generator and load in [3]. However, there were the following shortcomings: 1) Lack of theoretical proofs about the stability and convergence of the frequency synchronization point; 2) The coupling weights of each edge and damping constant of each node is unnecessarily assumed to be equal; 3) The analysis complexity increases with the presence of higher order derivatives; 4) The model does not take RES nodes into account. The non-uniform Kuramoto model and the multi-agent consensus protocol were used to derive the sufficient condition for the frequency synchronization of power grid in [4]. However, the frequency stability analysis of power grid in [4] only considered the network connectivity and the initial phase of the nodes, without taking into account the effect of the Laplacian matrix on the stability. The critical coupling strength for frequency synchronization in power grid was obtained in [5] and [6] in 2012 by assuming that the coupling weights of each edge and the damping constant of each node are equal and did not consider the RES nodes as well ${ }^{[5][6]}$, which was not agreeable with practical grids. The output of the generation nodes were controlled cooperatively according to the information exchanged with the ambient agent and the multi-agent consensus protocol, in order to guarantee the frequency stability of the grid ${ }^{[7]}$. This article considered the dynamics of renewable energy equipped with droop controller, but did not consider the storage nodes with different working states. A necessary and sufficient condition for the existence of frequency synchronization solution in micro-grid with droop-controlled inverters were derived in [8]. However, all loads were assumed to be constant power loads in [8]. The methods of analyzing power grid frequency synchronization are mainly divided into two approaches: the grid topology and the grid dynamics. From the grid topology approach, the synchronous ability of the various grid sizes and the evolution patterns are primarily compared and analyzed using numerical experiments and statistical physics methods [9] [10], which demonstrates the static characteristics in an analytical way, but cannot reflect the dynamical behavior of the power grid. From the grid dynamics approach, the relationship between dynamical behavior and the network parameters of the power 
grid, and the grid frequency synchronization conditions are obtained in an analytical way ${ }^{[3]}$.

In this paper, to analyze frequency stability from the dynamics approach, we establish an unified dynamical model for the power grid including RES nodes, which simplifies the analysis complexity about the power grid frequency synchronization. We show that the frequency stability of power grid is influenced not only by the network connectivity and the initial phase of the nodes, but also by the time-varying Laplacian matrix of the power grid. Reference [4] gives the condition for the existence of frequency synchronized solution, but the result cannot guarantee the stability of the synchronized solution. A supplementary condition is given in this paper to decide whether the synchronized solution is stable by estimating the eigenvalues of the time-varying Laplacian matrix. Thereby, we derive the sufficient conditions for frequency synchronization and its stability in power grids that contains RES nodes. Finally, the conditions are verified by the Western System Coordinating Council model and the Shaanxi North Power Grid.

The remainder of this paper is organized as follows: in Section 2, some relevant preliminaries and notations are introduced. In Section 3, the uniform mathematical power grid model for four different types of nodes are given. In Section 4, the sufficient condition for frequency synchronization and stability of power grid$\mathrm{s}$ are derived. The simulation results of both the Western System Coordinating Council and the Shaanxi North Power Grid are given in Section 5. Finally, some conclusions are drawn in Section 6.

\section{Preliminaries and notation}

\subsection{The set of phase differences and positive invariance}

From graphics geometry theory, $S^{1}=(-\pi,+\pi]$ denotes the unit circle and the $n$-torus $T^{n}=S^{1} \times S^{2} \ldots \times S^{n}$ is the Cartesian product of $n$ unit circles. For $\gamma \in[0, \pi]$, let $\Delta(\gamma) \subset T^{n}$ be a set of angle arrays $\left(\theta_{1}, \theta_{2}, \ldots, \theta_{n}\right) \in T^{n}$, so that there exists an arc of length $\gamma$ containing all $\theta_{1}, \theta_{2}, \ldots, \theta_{n}$ in its interior, which denotes the distance between two arbitrary angles in the phase arrays. Thus, an array of angles $\theta \in \Delta(\gamma)$ satisfies $\max _{i, j \in\{1,2, \ldots, n\}}\left|\theta_{i}-\theta_{j}\right| \leqslant \gamma$, i.e., at each time $t$ there exists an arc of length $\gamma$ containing all angles $\theta_{i}(t)$; the set of phase differences $\Delta(\gamma)$ is positively invariant if it is no longer increasing. ${ }^{[3]}$

\subsection{Algebraic graph theory}

An arbitrary complex network can be represented by the relevant weighted graph. ${ }^{[11]}$ A weighted directed graph is a triple set $G=(v, \varepsilon, A)$, where $v=$ 
$\{1,2, \ldots, n\}$ is the set of nodes, $\varepsilon=\{(i, j) \mid i, j \in v\}$ is the set of directed edges, and $\mathbf{A} \in R^{n \times n}$ is the adjacent matrix, $\mathbf{A}$ satisfies $a_{i j}>0$ for each directed edge $\{i, j\} \in \varepsilon$ and otherwise $a_{i j}=0 . a_{i j}$ is the coupling strength between node $i$ and $j$. Degree matrix $\mathbf{D}_{\mathbf{e}} \in R^{v \times v}$ is a diagonal matrix whose main diagonal entries are $\sum_{j=1}^{n} a_{i j}$. The node-edge incidence matrix $\mathbf{B} \in R^{v \times \varepsilon}$ is defined component-wise as $B_{k l}=1$ if node $k$ is the sink node of edge $l$ and as $B_{k l}=-1$ if node $k$ is the source node of edge $l$, with all other elements being zeros. Then the Laplacian matrix is given by $\mathbf{L}=\mathbf{D}_{\mathbf{e}}-\mathbf{A}$, and is positive semidefinite with eigenvalues satisfying $0=\lambda_{1}<\lambda_{2}<\ldots \lambda_{|v|}$, if $a_{i j}>0 .^{[12]}$

\subsection{Linear time-varying consensus protocol of multi-agent system}

Lemma 1. ${ }^{[13]}$ Consider $n$ nodes multi-agent system defined on a graph $G=$ $(v, \varepsilon, \mathbf{A}), x_{i}$ is the state variable of agent $i,\left(A_{k l}\right)=\alpha_{k l}$ is the coupling strength, which describes the interaction between agents, assume that each node satisfies the following equation,

$$
\dot{x}_{k}(t)=\sum_{l=1 . l \neq k}^{n} a_{k l}(t)\left(x_{l}(t)-x_{k}(t)\right),
$$

which is given by

$$
\dot{\mathbf{x}}=\mathbf{A}(t) \mathbf{x} .
$$

Assume that all but one of the eigenvalues of the system matrix $\mathbf{A}$ have strictly negative real part, the only exception is the trivial eigenvalues at zero, if and only if the associated digraph has (at least) one node from which all other nodes may be reached, then all the system state variables stably converge to a common value.

According to Lemma 1, we learn that, with the network connectivity varying, the eigenvalues of $\mathbf{A}(t)$ are also time-varying. When any, but zero eigenvalues of the system matrix $\mathbf{A}$, do not have strictly negative real part, or the globally reached node does not exist, then the equilibria states of Eq. (2) are no longer stable.

\section{Mathematical model of different nodes in a power grid}

\subsection{The dynamical model of the generator}

The dynamical model of the generator is given as follows

$$
D_{i} \dot{\theta}_{i}=P_{m i}-\left(E_{i}^{2} G_{i i}+\sum_{j=1}^{n} E_{i} E_{j} Y_{i j} \sin \left(\theta_{i}-\theta_{j}+\varphi_{i j}\right)\right),
$$


where, in terms of node $i, D_{i}$ and $M_{i}$ are the damping and inertia constants, respectively, $\theta_{i}$ of node $i$ is the angle of the shaft in radians with respect to a synchronously rotating reference, $\varphi_{i j}$ is the phase shifts caused by transfer conductance, and $E_{i}$ is the voltage magnitude of the synchronous machine. $Y_{i j}$ is the admittance of transmission line between node $i$ and node $j$, and $G_{i i}$ is the self-conductance of node $i$. More details about the model parameters and derivation of Eq. (3) could be found in Appendix A.

\subsection{The dynamical node of the load}

Similar to the generator, the dynamics of the load is given as follows

$$
M_{i} \ddot{\theta}_{i}=-P_{L i}-D_{i} \dot{\theta}_{i}-\left(E_{i}^{2} G_{i i}+\sum_{j=1}^{n} E_{i} E_{j} \sin \left(\theta_{i}-\theta_{j}\right)\right) .
$$

Note that, the main difference between (4) and (A. 29) in Appendix A is the mechanical power. Since the load node is the energy consumption node, $P_{L i}$ represents the active power that the load node consumes.

Similarly, when we investigate frequency synchronization, the inertia term can be omitted due to the fact that it only affects the convergence time of synchronization, thus dynamic models of the load nodes can be rewritten as

$$
D_{i} \dot{\theta}_{i}=-P_{L i}-\left(E_{i}^{2} G_{i i}+\sum_{j=1}^{n} E_{i} E_{j} \sin \left(\theta_{i}-\theta_{j}\right)\right) .
$$

\subsection{Model of RES nodes based on droop control}

For grid-connected RES nodes, it is very necessary to convert the DC or the non-industrial frequency AC power to the industrial frequency AC power by means of inverters. Power grid typically contains a bank of RES nodes equipped with power-frequency droop inverter controllers operated in parallel. Therefore, we establish the dynamics of RES nodes based on the droop control.

Droop control is a basic way of grid connected converters control, and is widely applied in the RES batteries converter technique ${ }^{[15]}$. The frequency droop control formula is

$$
f_{i}=f_{0_{i}}-n_{p_{i}} P_{i},
$$

where the parameter $n_{p_{i}}=X_{i} / 2 \pi E_{i} E$ is referred to as the droop coefficient, $E_{i}$ is the output voltage of inverter connected to the RES nodes, $E$ is the voltage of DC bus, $j X_{i}$ is the line impedance between inverter and bus, $f_{0_{i}}$ is the output frequency 
of the unload inverter, and $P_{i}$ is the output active power of the RES node. Proofs about droop control can be found in Appendix B. For simplicity, let $D_{i}=1 / n_{p_{i}}$, Eq. (6) is rewritten as

$$
D_{i} f_{i}=D_{i} f_{0_{i}}-P_{i} .
$$

In addition, we denote $f_{i}$ by $\dot{\theta}_{i}$, the nominal power $D_{i} f_{0_{i}}$ by $P_{d i}$, and the output power $P_{i}$ as is done in (A. 26) in Appendix A. Then Eq. (7) is rewritten as

$$
D_{i} \dot{\theta}_{i}=P_{d i}-\left(E_{i}^{2} G_{i i}+\sum_{j=1}^{n} E_{i} E_{j} \sin \left(\theta_{i}-\theta_{j}\right)\right) .
$$

Equation (8) is the model of RES nodes for discharging state.

The storage node works not only in discharge state, but also in charge state that can be treated as a load node, Thus, its model can be described by (5).

\section{Frequency synchronization and stability analysis of a power grid based on complex network theory}

We denote $G=(v, \varepsilon, \mathbf{A})$ as the topology of a power grid, where $v$ is the set of nodes, $\varepsilon$ is the set of transmission lines and $\mathbf{A}$ is the adjacency matrix. The power grid includes $n$ nodes and $m$ transmission lines denoted as $|v|=n,|\varepsilon|=m$. Then all the nodes can be denoted as $\left\{v_{G}, v_{L}, v_{D}\right\}$, where $v_{G}$ represents the set of the generator nodes, $v_{L}$ represents the set of the load nodes, and $v_{D}$ represents the set of the RES nodes.

In order to describe the various types of grid nodes using a uniform matrix form, we make the following transformation

$$
\begin{aligned}
& P_{m i}^{*}=P_{m i}-E_{i}^{2} G_{i i}, i \in v_{G}, \\
& P_{L i}^{*}=-P_{L i}-E_{i}^{2} G_{i i}, i \in v_{L}, \\
& P_{D i}^{*}= \pm P_{D i}-E_{i}^{2} G_{i i}, i \in v_{D},
\end{aligned}
$$

Then Eqs. (3), (5) and (8) can be written as

$$
\begin{aligned}
& D_{i} \dot{\theta}_{i}=P_{m i}^{*}-\sum_{j=1}^{n} E_{i} E_{j}\left|Y_{i j}\right| \sin \left(\theta_{i}-\theta_{j}\right), i \in v_{G}, \\
& D_{i} \dot{\theta}_{i}=P_{L i}^{*}-\sum_{j=1}^{n} E_{i} E_{j}\left|Y_{i j}\right| \sin \left(\theta_{i}-\theta_{j}\right), i \in v_{L}, \\
& D_{i} \dot{\theta}_{i}=P_{D i}^{*}-\sum_{j=1}^{n} E_{i} E_{j}\left|Y_{i j}\right| \sin \left(\theta_{i}-\theta_{j}\right), i \in v_{D},
\end{aligned}
$$


Define

$$
a_{i j} \triangleq E_{i} E_{j}\left|Y_{i j}\right|,\{i, j\} \in \varepsilon,
$$

so we can rewrite the model in Eq. (10) in the following form

$$
D_{i} \dot{\theta}_{i}=P_{i}^{*}-\sum_{j=1}^{n} a_{i j} \sin \left(\theta_{i}-\theta_{j}\right), i \in v,
$$

The above equation is described by using the following Laplacian matrix notation,

$$
\mathbf{D} \dot{\theta}=\mathbf{P}^{*}-\mathbf{B W} \sin \left(\mathbf{B}^{\mathbf{T}} \theta\right),
$$

where $\theta=\left[\theta_{1}, \ldots, \theta_{n}\right]^{T}, \mathbf{D}=\left[D_{1}, \ldots, D_{n}\right]^{T}, \mathbf{P}^{*}=\left[P_{m 1}^{*}, \ldots, P_{\left|v_{G}\right|}^{*}, P_{L 1}^{*}, \ldots, P_{\left|v_{L}\right|}^{*}, P_{D 1}^{*}, \ldots\right.$, $\left.P_{\left|v_{D}\right|}^{*}\right]^{T}, \mathbf{B}$ and $\mathbf{W}$ are the incidence matrix and weighted matrix, respectively.

To achieve a stable frequency synchronization solution in a power grid, firstly, it is necessary for the synchronization solution of Eq. (13) to exist, and secondly, the synchronization solution needs to guarantee to be stable.

A solution $\theta$ of Eq. (12) is said to be synchronized if $\dot{\theta}_{i}=\dot{\theta}_{j}$ for each node, so the frequency difference between two arbitrary nodes can be represented as follows

$$
\dot{\theta}_{i}-\dot{\theta}_{j}=\frac{P_{i}^{*}}{D_{i}}-\frac{P_{j}^{*}}{D_{j}}-\sum_{k=1}^{n}\left(\frac{a_{i k}}{D_{i}} \sin \left(\theta_{i}-\theta_{k}\right)-\frac{a_{j k}}{D_{j}} \sin \left(\theta_{j}-\theta_{k}\right)\right), i, j \in v .
$$

To achieve frequency synchronization in power grid, the coupling strength between nodes $i$ and $j$ has to dominate the networks non-uniformity as shown in Eq. (15)

$$
\frac{P_{i}^{*}}{D_{i}}-\frac{P_{j}^{*}}{D_{j}}<\sum_{k=1}^{n}\left(\frac{a_{i k}}{D_{i}} \sin \left(\theta_{i}-\theta_{k}\right)-\frac{a_{j k}}{D_{j}} \sin \left(\theta_{j}-\theta_{k}\right)\right), i, j \in v .
$$

By a scale transformation of inequalities (15), we get

$$
\Gamma_{\min } \sin (\gamma) \geqslant \Gamma_{\text {critical }},
$$

where $\Gamma_{\min }=n \min _{i \neq j}\left\{\frac{a_{i j}}{D_{i}}\right\}, \Gamma_{\text {critical }}=\max _{i \neq j}\left|\frac{P_{i}^{*}}{D_{i}}-\frac{P_{j}^{*}}{D_{j}}\right|$. There exists the frequency synchronized solution if the inequalities in Eq. (16) is satisfied.

The synchronization frequency can be obtained by summing all the rows of the matrix equation, Eq. (13), such that

$$
\sum_{i=1}^{n} D_{i} \dot{\theta}_{i}=\sum_{i=1}^{n} P_{i}^{*}+\sum_{i=1}^{n} \sum_{j=1}^{n} a_{i j} \sin \left(\theta_{i}-\theta_{j}\right)
$$


Assuming that there is the synchronization frequency $\omega_{\text {sync }}$, the non-symmetric property of the sine function implies that the last term $\sum_{i=1}^{n} \sum_{j=1}^{n} a_{i j} \sin \left(\theta_{i}-\theta_{j}\right)$ is zero, then, $\omega_{\text {sync }}=\sum_{i=1}^{n} P_{i}^{*} \mid \sum_{i=1}^{n} D_{i}$.

After obtaining the synchronization solution, we need to analyze the stability of this solution. To prove the stability of the synchronized solution of Eq. (13), we consider the derivative of Eq. (12)

$$
\ddot{\theta}_{i}=-\sum_{j=1}^{n} \frac{a_{i j}}{D_{i}} \cos \left(\theta_{i}-\theta_{j}\right)\left(\dot{\theta}_{i}-\dot{\theta}_{j}\right)
$$

By defining $\alpha_{i j}{ }^{*}=\frac{a_{i j}}{D_{i}} \cos \left(\theta_{i}-\theta_{j}\right)$, we rewrite Eq. (18) as

$$
\ddot{\theta}_{i}=-\sum_{j=1}^{n} \alpha_{i j}{ }^{*}\left(\dot{\theta}_{i}-\dot{\theta}_{j}\right)
$$

The Laplacian matrix form of Eq. (19) is

$$
\ddot{\theta}=-\mathbf{L}(\mathbf{t}) \dot{\theta}
$$

where $\mathbf{L}(t)=\operatorname{diag}\left(\sum_{j=1}^{n} \alpha_{i j}{ }^{*}\right)-\left\{\alpha_{i j}{ }^{*}\right\}$ is the Laplacian matrix, $\operatorname{diag}\left(\sum_{j=1}^{n} \alpha_{i j}{ }^{*}\right)$ represents a diagonal matrix whose main diagonal entries are $\sum_{j=1}^{n} \alpha_{i j}{ }^{*}$, and $\left\{\alpha_{i j}{ }^{*}\right\}$ represents a matrix with all entries being $\alpha_{i j}{ }^{*}$.

Obviously the form of Eq. (20) is similar to the form of the consensus protocol of the linear time invariant multi-agent system shown in Eq.(2). So we use Lemma 1 to analyze the stability of the frequency synchronization solution of a power grid.

Theorem 1. Consider the power system (13), if $\theta(0) \in \Delta(\gamma), \gamma \in\left[\gamma_{\min }, \pi / 2\right]$, then there exists the synchronization frequency, where $\gamma_{\min }=\arcsin \left(\Gamma_{\text {critical }} / \Gamma_{\min }\right)$. Furthermore, assuming that the network is globally reachable for all time and that all but one of the eigenvalues of the time-varying Laplacian matrix $-L(t)$ have strictly negative real part, the only exception is the trivial eigenvalue at zero, if $\Delta(\gamma)$ is positively invariant, and the synchronization frequency is stable, then the frequency $\dot{\theta}_{i}(t)$ exponentially converges to the following common value: $\dot{\theta}_{\infty}=$ $\omega_{\text {sync }}=\sum_{i=1}^{n} P_{i}^{*} \mid \sum_{i=1}^{n} D_{i}$ 
Proof: Note that

$$
\gamma_{\min }=\arcsin \left(\frac{\Gamma_{\text {critical }}}{\Gamma_{\min }}\right)
$$

Then,

$$
\sin \left(\gamma_{\min }\right)=\frac{\Gamma_{\text {critical }}}{\Gamma_{\min }}
$$

Since for $\gamma \in\left[\gamma_{\min }, \pi / 2\right], \sin (\gamma)$ is strictly monotonously increasing on the interval $[0, \pi / 2)$, then

$$
\sin (\gamma) \geqslant \sin \left(\gamma_{\min }\right)=\frac{\Gamma_{\text {critical }}}{\Gamma_{\min }}
$$

Due to $\Gamma_{\min }>\Gamma_{\text {critical }}>0$, Eq. (16) holds from the fact that it can be derived by multiplying both sides of Eq. (23) by $\Gamma_{\min }$, consequently, there exists a synchronization frequency.

If all but one of the eigenvalues of the time-varying Laplacian matrix $-\mathbf{L}(t)$ of Eq. (20) have strictly negative real part, and the only exception is the trivial eigenvalues at zero, we have

$$
-\mathbf{L}(t)=\left\{\begin{array}{l}
-\sum_{j=1, j \neq i}^{n} \frac{a_{i j}}{D_{i}} \cos \left(\theta_{i}-\theta_{j}\right), i=j \\
\frac{a_{i j}}{D_{i}} \cos \left(\theta_{i}-\theta_{j}\right), i \neq j .
\end{array}\right.
$$

Therefore, $-\mathbf{L}(t)$ must be a Metzler matrix with row sum being zero (i.e, $-\mathbf{L}(t)$ is the matrix whose all main diagonal elements are negative and all off-diagonal elements are non-negative). According to Eq. (21), for $t>0$, the following inequalities holds

$$
\left\{\begin{array}{l}
-\sum_{j=1, j \neq i}^{n} \frac{a_{i j}}{D_{i}} \cos \left(\theta_{i}-\theta_{j}\right) \leqslant 0 \\
\frac{a_{i j}}{D_{i}} \cos \left(\theta_{i}-\theta_{j}\right) \geqslant 0
\end{array}\right.
$$

Because of $\frac{a_{i j}}{D_{i}} \geqslant 0, \forall i, j$, then the term $\cos \left(\theta_{i}-\theta_{j}\right) \geqslant 0, \forall i, j$ is true, that is $\gamma \in\left[\gamma_{\min }, \pi / 2\right],\left|\theta_{i}-\theta_{j}\right| \leqslant \gamma$ for $t>0$, so the set $\Delta(\gamma)$ is positively invariant, and the synchronization frequency is stable.

The conditions for the existence of synchronization solution given in [3] is described as follows: $\gamma \in\left[\gamma_{\min }, \pi / 2\right], \gamma_{\min }=\arcsin \left(\Gamma_{\text {critical }} / \Gamma_{\min }\right)$, then the set $\Delta(\gamma)$ 
is positively invariant. However, Theorem 1 in this work shows that, when the conditions given in [3] are satisfied, the existence of synchronization solution of the system can be proved, but the stability of this solution cannot be guaranteed. In this paper, assuming that the network is globally reachable for all time and that all but one of the eigenvalues of the time-varying Laplacian matrix $-\mathbf{L}(t)$ have strictly negative real part, the only exception is the trivial eigenvalues at zero; therefore, the set $\Delta(\gamma)$ is positively invariant, and the synchronization frequency is stable. Our Theorem in this paper gives supplementary information for the stability of the the frequency synchronization solution. The following simulation examples validate the applicability of this Theorem.

\section{Model simulation and analysis}

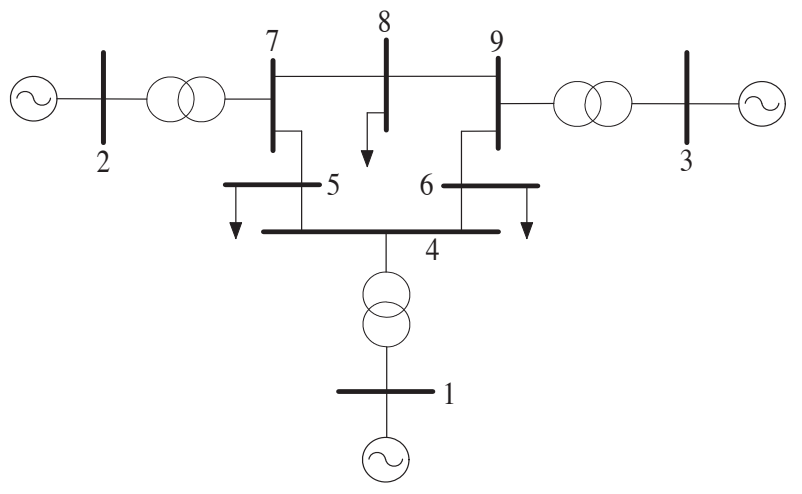

Figure 1. The grid topology of the Western System Coordinating Council

The grid topology of the Western System Coordinating Council (WSCC 9BUS $)^{[14]}$ is shown in Fig. 1, where buses 1, 2 and 3 connected to generators is treated as generator nodes, buses 5, 6 and 8 connected to loads can be viewed as load nodes, buses 4, 7 and 9 are transformer buses, and can be treated as single paths. We thus obtain a 6-node network model. Additionally, we assume that there are three generator nodes, where node 1 is the classical generator node, node 2 is the renewable energy node, and node 3 is the storage node that operates in discharging state. The nodes parameters and lines parameters of the Western System Coordinating Council in the simulations are given in Tables 1 and 2. From Table 2, the branch resistance value is an order of magnitude smaller than the branch reactance value. 
Table 1: The nodes parameters of the Western System Coordinating Council

\begin{tabular}{|c|c|c|c|c|}
\hline \multirow{2}{*}{ Node Number } & \multicolumn{2}{|c|}{ Node Voltage } & Node Power & Node Damping \\
\cline { 2 - 5 } & $U(p . u)$. & $\delta\left({ }^{\circ}\right)$ & $P(p . u)$. & $D(p . u)$. \\
\hline 1 (Bus 1) & 1.0400 & 0 & 16.30 & 0.2 \\
\hline 2 (Bus 2) & 1.0250 & 9.2800 & 8.00 & 0.1 \\
\hline 3 (Bus 3) & 1.0250 & 4.6648 & 0.85 & 0.1 \\
\hline 4 (Bus 4) & 0.9956 & -3.9888 & -1.00 & 0.1 \\
\hline 5 (Bus 5) & 1.0127 & -3.6874 & -0.90 & 0.1 \\
\hline 6 (Bus 6) & 1.0159 & 0.7275 & -0.80 & 0.1 \\
\hline
\end{tabular}

Table 2: The line parameters of the Western System Coordinating Council

\begin{tabular}{|c|c|c|c|}
\hline Source Node & Sink Node & Branch Resistance & Branch Reactance \\
\hline 1 & 4 & 0.0100 & 0.2034 \\
\hline 1 & 5 & 0.0170 & 0.2104 \\
\hline 2 & 4 & 0.0320 & 0.3433 \\
\hline 2 & 6 & 0.0085 & 0.2543 \\
\hline 3 & 5 & 0.0390 & 0.4099 \\
\hline 3 & 6 & 0.0119 & 0.2708 \\
\hline
\end{tabular}

We can calculate from the parameters to obtain: $\Gamma_{\min }=n \min _{i \neq j}\left\{\frac{a_{i j}}{D_{i}}\right\}=598.9573$, $\Gamma_{\text {critical }}=\max _{i \neq j}\left|\frac{P_{i}^{*}}{D_{i}}-\frac{P_{j}^{*}}{D_{j}}\right|=91.5, \gamma_{\min }=\arcsin \left(\frac{\Gamma_{\text {critical }}}{\Gamma_{\min }}\right)=8.7872^{\circ}$ and $\gamma=\max _{i \neq j} \mid \theta_{i}(0)-$ $\theta_{j}(0) \mid=13.269^{\circ}$. Since $\gamma \in\left(\gamma_{\min }, \pi / 2\right)$, according to Theorem 1 , there exists the frequency synchronized solution, since Laplace matrix $\mathbf{L}(t)$ is time-varying, its eigenvalues change as well, where $\alpha_{i j}{ }^{*}=\frac{a_{i j}}{D_{i}} \cos \left(\theta_{i}-\theta_{j}\right)$. Figure 2 (b) illustrates that the largest eigenvalue of $-\mathbf{L}(t)$ is zero before 9 seconds, after 9 seconds, the maximum eigenvalue of the system is far greater than zero, which does not satisfy the stability condition given by Theorem 1. Figure 2 (c) illustrates the phase differences divergence. Therefore, although there exists a frequency synchronization solution, the solution is unstable. Thus the system does not obtain the stable synchronization frequency as shown in Figure 2 (a).

In Figure 3 the simulation parameters are changed. The damping vector is $\mathbf{D}=$ $[2,1,1,1,1,1]^{T}$. The injection power $\mathbf{P}=[1.63,0.8,0.85,-1,-0.9,-0.8]^{T}$. The simulation results are shown in Figure 3 . In this case we have $\Gamma_{\min }=n \min _{i \neq j}\left\{\frac{a_{i j}}{D_{i}}\right\}=$ 59.986, $\Gamma_{\text {critical }}=\max _{i \neq j}\left|\frac{P_{i}^{*}}{D_{i}}-\frac{P_{j}^{*}}{D_{j}}\right|=1.85, \gamma_{\min }=\arcsin \left(\frac{\Gamma_{\text {critical }}}{\Gamma_{\min }}\right)=1.77^{\circ}$ and $\gamma$ is the 


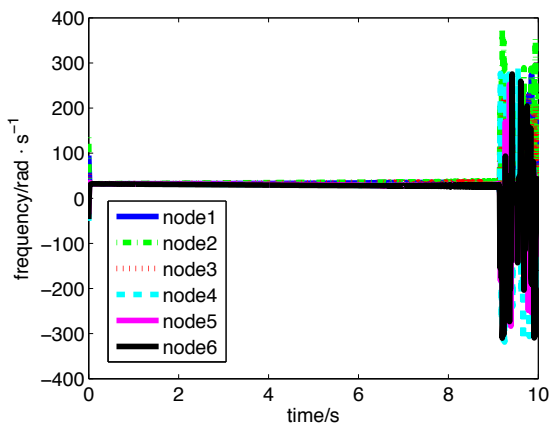

(a) The nodes frequency variation
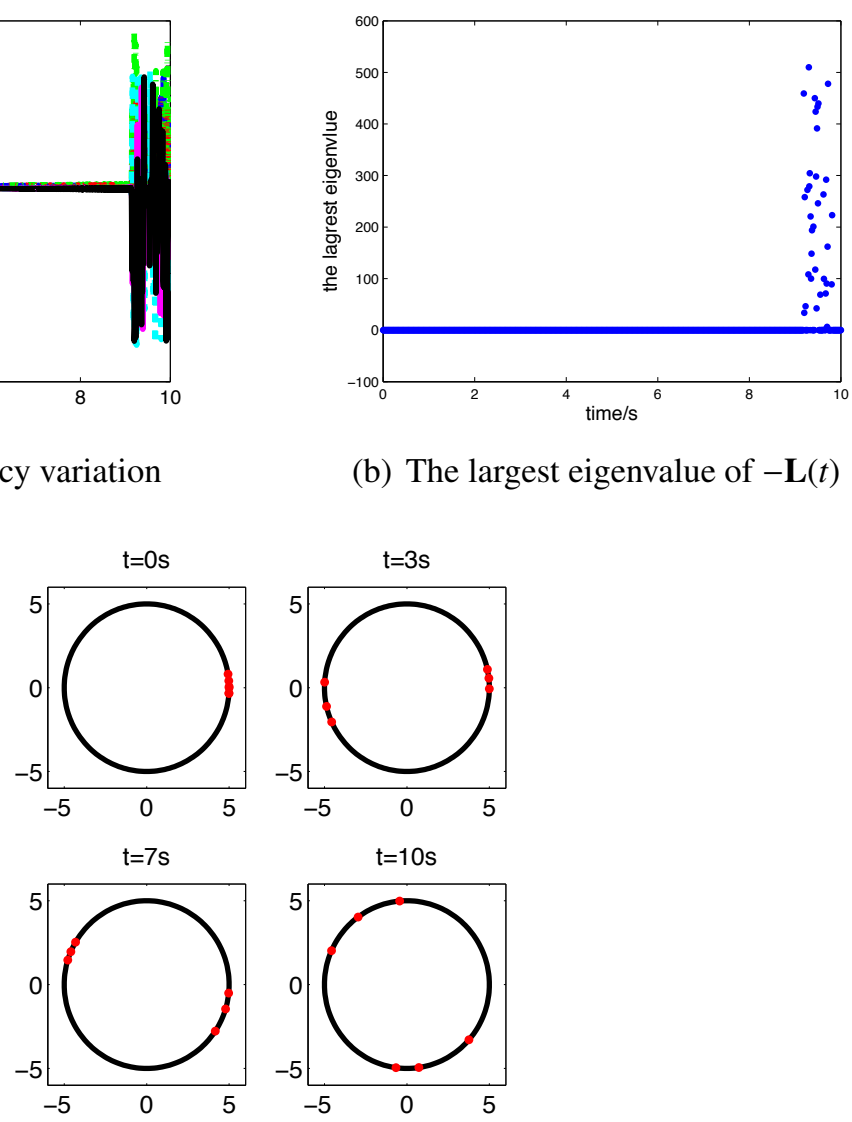

(b) The largest eigenvalue of $-\mathbf{L}(t)$
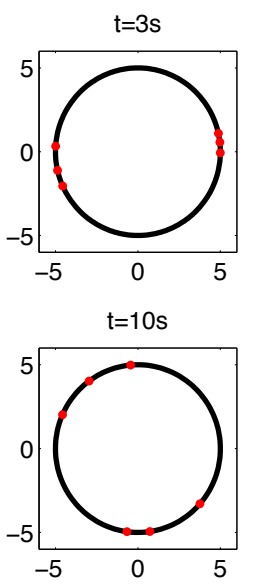

(c) Node phase variation

Figure 2. Subplots (a), (b), (c) illustrate the nodes frequency variation, the largest eigenvalue of $-\mathbf{L}(t)$ and the node phase variation of the Western System Coordinating Council, respectively. Simulation results using $\mathbf{D}=$ $[0.2,0.1,0.1,0.1,0.1,0.1]^{T}$ and $\mathbf{P}=[16.3,8,0.85,-1,-0.9,-0.8]^{T}$.

same as that in Figure 2.

For $\gamma \in\left(\gamma_{\min }, \pi / 2\right)$, there exists a frequency synchronization solution. Moreover, the largest eigenvalue of $-\mathbf{L}(t)$ is always zero and others are smaller than zero, which satisfies the stability condition given by Theorem 1 , the set $\Delta(\gamma)$ is positively invariant. Fig. 3 (c) illustrates that the phase difference between arbitrary two nodes converges, that is, the synchronization frequency is stable for these parameters. Therefore, the solutions of the system exponentially converge to 


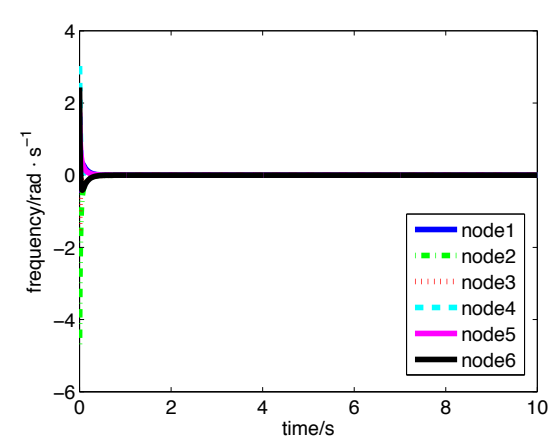

(a) The nodes frequency variation
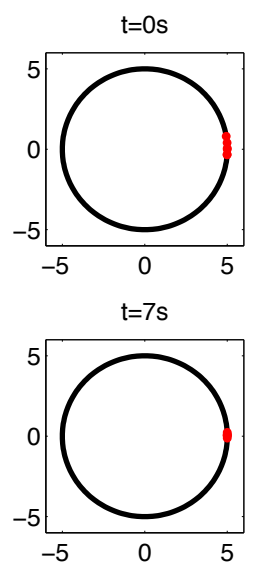

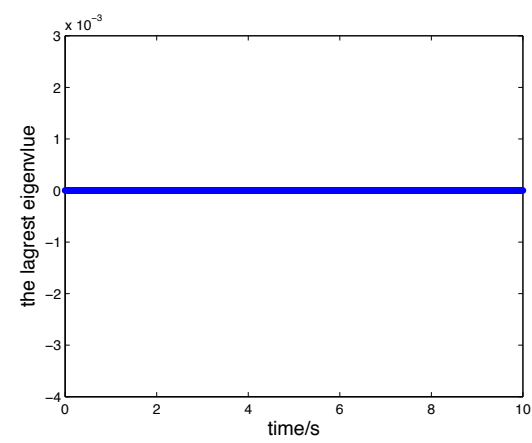

(b) The largest eigenvalue of $-\mathbf{L}(t)$
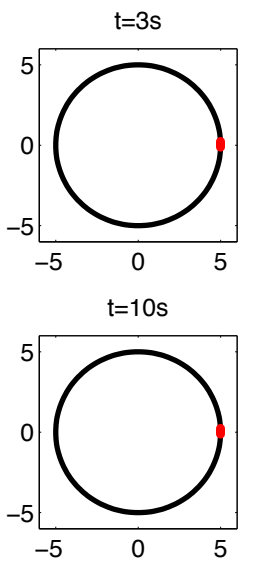

(c) Node phase variation

Figure 3. Subplots (a), (b), (c) illustrate the nodes frequency variation, the largest eigenvalue of $-\mathbf{L}(t)$ and the node phase variation of Western System Coordinating Council, respectively. Simulation results using $\mathbf{D}=[2,1,1,1,1,1]^{T}$ and $\mathbf{P}=$ $[1.63,0.8,0.85,-1,-0.9,-0.8]^{T}$.

stable synchronization frequency in Figure 3 (a). In another words, the frequency of all nodes converge to the stable value $\dot{\theta}_{\infty}=\sum_{i=1}^{n} P_{i}^{*} \mid \sum_{i=1}^{n} D_{i}$.

The North Local Power Grid of Shannxi Province with 58 generator nodes and 57 load nodes is used to verify Theorem 1 in this paper. The nodes data and branch data can be found in Appendix C, where the node type 1 (node type 2) means the node is a generator (a load). We can calculate from the parameters in Appendix C 
and obtain $\Gamma_{\min }=n \min _{i \neq j}\left\{\frac{a_{i j}}{D_{i}}\right\}=11.0764, \Gamma_{\text {critical }}=\max _{i \neq j}\left|\frac{P_{i}^{*}}{D_{i}}-\frac{P_{j}^{*}}{D_{j}}\right|=4.3505, \gamma_{\min }=$ $\arcsin \left(\frac{\Gamma_{\text {critical }}}{\Gamma_{\text {min }}}\right)=23.1246^{\circ}$ and $\gamma=\max _{i \neq j}\left|\theta_{i}(0)-\theta_{j}(0)\right|=60.2112^{\circ}$. Obviously, for $\gamma \in\left(\gamma_{\min }, \pi / 2\right)$, there exists a frequency synchronization solution. Moreover, as shown in Figure $4(\mathrm{~b})$, the largest eigenvalue of $-\mathbf{L}(t)$ is always zero and other eigenvalues are smaller than zero, which satisfies the stability condition given in Theorem 1, the set $\Delta(\gamma)$ is positively invariant. Figure 4 (c) illustrates that the phase difference between arbitrary two nodes converges, that is the synchronization frequency is stable under these parameters. Therefore, the solutions of the system exponentially converge to stable synchronization frequency as shown in Figure 4 (a). In Figure 5, the simulation parameters are changed.

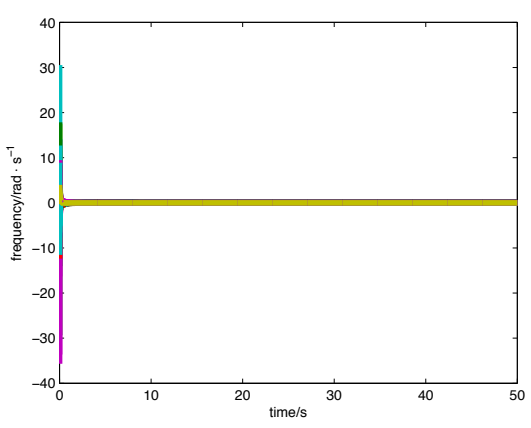

(a) The nodes frequency variation
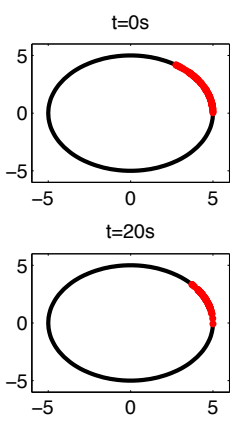

(c) Node phase variation

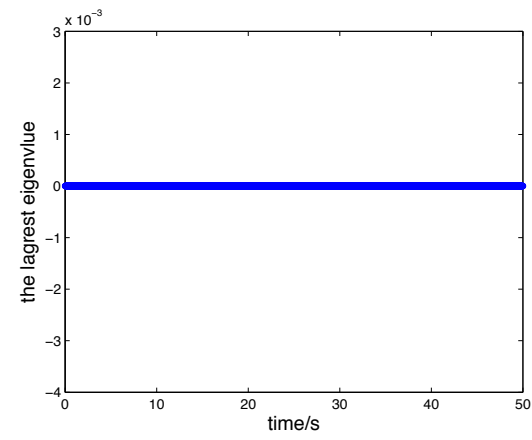

(b) The largest eigenvalue of $-\mathbf{L}(t)$
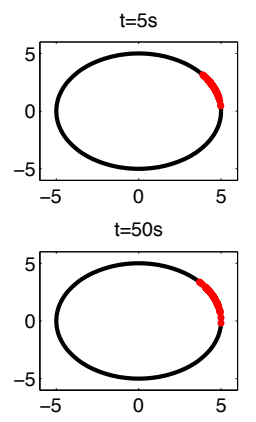

Figure 4. Subplots (a), (b), (c) illustrate the nodes frequency variation, the largest eigenvalue of $-\mathbf{L}(t)$ and the node phase variation of the North Local Power Grid of Shannxi Province, respectively. Simulation results using the parameters in Appendix C 
The damping of all nodes are the same as those in Appendix C, but the power of all the nodes are 5 times larger than those in Appendix C. In this case we have $\Gamma_{\min }=n \min _{i \neq j}\left\{\frac{a_{i j}}{D_{i}}\right\}=11.0764, \Gamma_{\text {critical }}=\max _{i \neq j}\left|\frac{P_{i}^{*}}{D_{i}}-\frac{P_{j}^{*}}{D_{j}}\right|=8.8901, \gamma_{\min }=$ $\arcsin \left(\frac{\Gamma_{\text {critical }}}{\Gamma_{\min }}\right)=53.3825^{\circ}$ and $\gamma=\max _{i \neq j}\left|\theta_{i}(0)-\theta_{j}(0)\right|=60.2112^{\circ}$.According to Theorem1, there exists the frequency synchronization solution, since Laplace ma$\operatorname{trix} \mathbf{L}(t)=\operatorname{diag}\left(\sum_{j=1}^{n} \alpha_{i j}{ }^{*}\right)-\left\{\alpha_{i j}{ }^{*}\right\}$ is time-varying, its eigenvalues change as well, where $\alpha_{i j}{ }^{*}=\frac{a_{i j}}{D_{i}} \cos \left(\theta_{i}-\theta_{j}\right)$. Figure 5 (b) illustrates that the largest eigenvalue of $-\mathbf{L}(t)$ is zero before 4.8 seconds, after 4.8 seconds the maximum eigenvalue of the system is greater than zero, which does not satisfy the stability condition given in Theorem 1. As shown in Figure 5 (c), the phase differences are divergent. Therefore, although there does exist the frequency synchronization solution, the solution is unstable. Thus the system does not obtain the stable synchronization frequency as shown in Figure 5 (a).

\section{Conclusions}

The models of generator and load nodes, according to rotor dynamics of the generator and the RES nodes and equipped with power-frequency droop inverter controllers, are given in this paper, where the storage nodes have two operating states. We proved that the stability of synchronization solutions depends on the Laplacian matrix $-\mathbf{L}(t)$ given in Eq. (20), using the consensus protocol of the linear time-varying multi-agent system. If all but one of the eigenvalues of the time-varying Laplacian matrix $-\mathbf{L}(t)$ have strictly negative real part, and the only exception is the trivial eigenvalue at zero, then $\Delta(\gamma)$ is positively invariant and the synchronization solutions is stable. Simulation examples validate the applicability of this Theorem using the power grid of the Western System Coordinating Council and the North Local Power Grid of Shannxi Province.

\section{Acknowledgement}

The work is supported in part by Key Program of Nature Science Fund of Shaanxi Province (2016ZDJC-01), IRT of Shaanxi Province (2013KCT-04). 


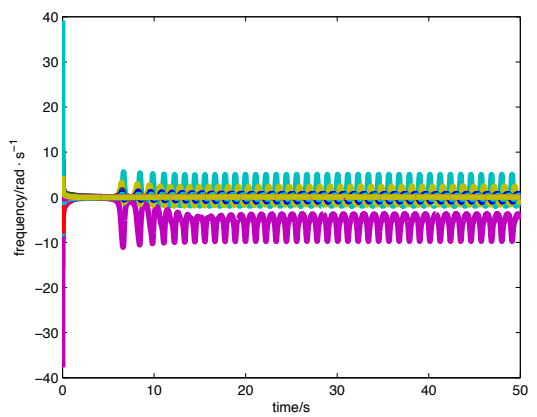

(a) The nodes frequency variation
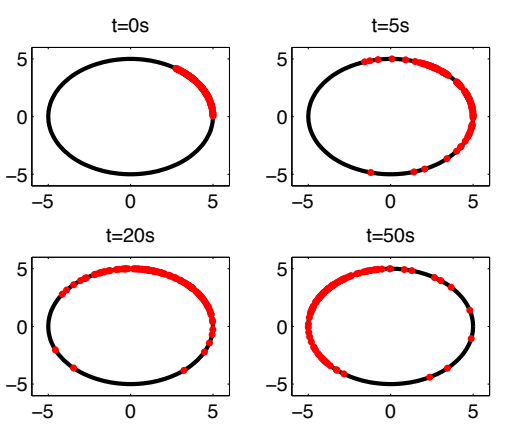

(c) Node phase variation

Figure 5. Subplots (a), (b), (c) illustrate the nodes frequency variation, the largest eigenvalue of $-\mathbf{L}(t)$ and node phase variation of the North Local Power Grid of Shannxi Province, respectively. Simulation results using the node power parameters which are 5 times larger than those in Appendix C

\section{Reference}

[1] C.C. Chu, H.C. Iu, Complex networks theory for modern smart grid spplications: a survey, J. IEEE Journal on Emerging \& Selected Topics in Circuits \& Systems 7(2) (2017) 177-191.

[2] F.A. Rodrigues, T.K.DM. Peron, P. Ji, J. Kurths, The Kuramoto model in complex networks, J. Physics Reports 610 (2016) 1-98.

[3] G. Filatrella, A.H. Nielsen, N.F. Pedersen, Analysis of a power grid using a Kuramoto-like model, J. European Physical Journal B 61(4) (2008) 485-491. 
[4] F. Dörfler, F. Bullo, Synchronization and transient stability in power networks and non-uniform Kuramoto oscillators, J. SIAM Journal on Control \& Optimization 50(3) (2012) 1616-1642.

[5] R. Carareto, M.S. Baptista, C. Grebogi, Natural synchronization in powergrids with anti-correlated units, J. Communications in Nonlinear Science \& Numerical Simulation 18(4) (2012) 1035-1046.

[6] S. Lozano, L. Buzna, A.D. Guilera, Role of network topology in the synchronization of power systems, J. The European Physical Journal B 231(85) (2012) 231-238.

[7] L.Y. Lu, C.C. Chu, Consensus-based secondary frequency and voltage droop control of virtual synchronous generators for isolated AC micro-grids, J. IEEE Journal on Emerging \& Selected Topics in Circuits \& Systems 5(3) (2015) 443-455.

[8] J.W.S. Porco, F. Dörfler, F. Bullo, Synchronization and power sharing for droop-controlled inverters in islanded microgrids, J. Automatica 49(9) (2013) 2603-2611.

[9] J. Wang, Y.H. Liu, Y.Jiao, H.Y. Hu, Cascading dynamics in congested complex networks, J. The European Physical Journal B 67(1) (2009) 95-100.

[10] L.X. Yang, J. Jiang, Impacts of link addition and removal on synchronization of an elementary power network, J. Physica A 479 (2017) 99-107.

[11] C. Godsil, Algebraic graph theory, J. Springer, 2001.

[12] F. Dörfler, F. Bullo, Kron, Reduction of graphs with applications to electrical networks, J. IEEE Transactions on Circuits \& Systems I: Regular Papers 60(1) (2013) 150-163.

[13] L. Moreau, Stability of continuous-time distributed consensus algorithms, J. IEEE Conference on Decision and Control 4 (2004) 3998-4003.

[14] P.M. Anderson, A.A. Fouad, Power system control and stability, J. Wiley, 2003.

[15] X.F. Sun, Y.C. Hao, Q.F. Wu, X.Q. Guo, B.C.Wang, A Multifunctional and wireless droop control for distributed energy storage units in islanded AC 
microgrid applications, J. IEEE Transactions on Power Electronics 32(1) (2017) 736-751.

\section{Appendix A.}

The motion equation of the generator rotor is governed by

$$
J \ddot{\delta}=T_{a},
$$

where $\delta$ is the mechanical angle of the shaft in radians with respect to a fixed reference, $J$ is the moment of inertia of the rotor in $\mathrm{kg} \cdot \mathrm{m}^{2}$, and $T_{a}$ is the sum torque in Newton meters.

$$
\begin{aligned}
& J \ddot{\delta}=T_{m}-T_{L} \\
& J \ddot{\delta}=T_{m}-\left(T_{e}+T_{d}\right)
\end{aligned}
$$

The angular reference is chosen with respect to a synchronously rotating reference frame, then

$$
\begin{gathered}
\quad \delta=\omega_{R} t+\theta_{m}, \\
\dot{\delta}=\omega_{R}+\dot{\theta}_{m}=\omega_{R}+\omega_{m} \\
\text { and } \ddot{\delta}=\ddot{\theta}_{m}=\dot{\omega}_{m},
\end{gathered}
$$

where $\theta_{m}$ is the mechanical angle of the shaft in radians with respect to a synchronously rotating reference frame, $\dot{\theta}_{m}$ is the angular velocity of the shaft, $\omega_{R}$ is synchronously rotating angular velocity.

Another form of (A.2) is obtained by multiplying its both sides with $\omega_{m}$, as shown by Eq. (A.5)

$$
\begin{aligned}
& J \omega_{m} \ddot{\theta}_{m}=P_{m}-P_{L} \\
& M \ddot{\theta}_{m}=P_{m}-P_{L},
\end{aligned}
$$

where $P_{m}=T_{m} \omega_{m}$ is the mechanical power, $P_{L}=T_{L} \omega_{m}$ is the load power. The quantity $J \omega_{m}$ is called the inertia constant and is denoted by $M$, it is related to kinetic energy of the rotating masses $W_{k}$, where $W_{k}=\frac{1}{2} J \omega_{m}^{2}$. Then $M$ can be calculated as follows

$$
M=J \omega_{m}=\frac{2 W_{k}}{\omega_{m}} .
$$


The electrical angle $\theta_{e}$ can be used to denote all of the equations above,

$$
\theta=\theta_{e}=\frac{p}{2} \theta_{m},
$$

where $p$ is the number of pole pairs of the (generator) motor, which is calculated as follows

$$
p=\frac{120 f_{R}}{n_{R}}
$$

where, $n_{R}$ is the rated shaft speed in $\mathrm{rad} / \mathrm{min}, f_{R}$ is the synchronously rotating frequency in $\mathrm{Hz}$.

The equation (A.1) can be equivalently written as

$$
\left(\frac{2 J}{p}\right) \ddot{\theta}=\left(\frac{2 J}{p}\right) \dot{\omega}=T_{a}(\mathrm{~N} \cdot \mathrm{m}) .
$$

Now, normalize Eq. (A.9) by dividing both sides by a constant equal to the rated torque at the rated speed

$$
T_{B}=\frac{S_{B 3}}{\omega_{m R}}=\frac{60 S_{B 3}}{2 \pi n_{R}},
$$

where $S_{B 3}$ is the rated three-phase power (VA), $\omega_{m R}=2 \pi n_{R} / 60$ is the rated angular velocity of the shaft.

According to Eqs (A.8)-(A.10), we have

$$
\left(\frac{J \pi^{2} n_{R}^{2}}{900 \omega_{R} S_{B 3}}\right) \dot{\omega}=\frac{T_{a}}{T_{B}}=T_{a u} \text { p.u., }
$$

where $\frac{T_{a}}{T_{B}}=T_{a u}$. The moment of inertia $J=\frac{746\left(W R^{2}\right)}{500 \mathrm{~g}}$ can be obtained by using the method in [14], and $\left(W R^{2}\right) / g$ is the unit of $J$. Then, by the following transformation

$$
\begin{gathered}
\frac{746\left(W R^{2}\right) \pi^{2} n_{R}^{2}}{550 g \times 900 \omega_{R} S_{B 3}} \dot{\omega}=T_{a u} \text { p.u. } \\
W_{k}=\frac{1}{2} J \omega_{m}^{2}=\frac{1}{2} \times \frac{746\left(W R^{2}\right)}{550 g} \times \frac{\left(2 \pi n_{R}\right)^{2}}{3600}=\frac{746\left(W R^{2}\right) \pi^{2} n_{R}^{2}}{550 g \times 1800} \\
H \triangleq \frac{W_{k}}{S_{B 3}}
\end{gathered}
$$

we have

$$
\left(\frac{2 W_{k}}{S_{B 3} \omega_{R}}\right) \dot{\omega}=T_{a u} \text { p.u.. }
$$




$$
\left(\frac{2 H}{\omega_{R}}\right) \dot{\omega}=T_{a u} \text { p.u.. }
$$

For a classical model of the synchronous machine, recognizing that the angular speed is nearly constant, the accelerating power $P_{a}$ in p.u. is nearly equal to the accelerating torque $T_{a}{ }^{[14]}$, then the generator becomes

$$
\left(\frac{2 H}{\omega_{R}}\right) \dot{\omega} \cong P_{a} \text { p.u.. }
$$

In addition, the damping torque (damping power) includes both the mechanical and electrical damping, which is represented by $D \omega$, then (A.17) can be rewritten as

$$
\left(\frac{2 H}{\omega_{R}}\right) \dot{\omega}=P_{m}-P_{e}-P_{d}=P_{m}-P_{e}-D \omega \mathrm{pu},
$$

where $P_{d}=D \omega$ is the damping power. The concrete formula of the electrical output power $P_{e}$ of the generator is derived as follows. Consider a power system consisting of one machine connected to an infinite bus through a transmission line. A schematic representation of this case is shown in Figure A. 1 (a), $E$ is the internal voltage of the synchronous machine, $L$ is the equivalent inductance of the transmission line, and $V$ is the voltage of the infinite bus, which is used as reference. The equivalent electrical circuit of the system is given in Figure A. 1 (b), $\mathbf{E}=E \angle \delta$ is the voltage vector of the synchronous machine and $\delta$ is the voltage phase, and $\mathbf{V}=V \angle 0$ is the voltage vector of the infinite bus, $x_{d}^{\prime}$ is the transient reactance of the machine, $\bar{V}_{t}$ is the terminal voltage of the synchronous machine, which can be eliminated by using $Y-\Delta$ transformation, $\bar{Z}_{s}$ is the equivalent shunt impedance at the machine terminal, and $\bar{Z}_{T L}$ is the series impedance of the transmission line. Another equivalent circuit of Figure 1 (b) is given in Figure A. 1 (c) by a $Y-\Delta$ transformation.

Assumptions for deriving the model are the following: 1) The mechanical power input remains constant during the transient period. 2) Damping or asynchronous power is negligible. 3) The synchronous machine can be represented by a constant voltage source and its mechanical angle coincides with the electrical phase angle of the voltage. 4) If a local load is fed at the terminal voltage of the machine, it can be represented by a constant impedance to neutral point.

In Figure A. 1 (a) we define: Nodes 0, 1 and 2 are the reference nodes, the internal voltage node of the synchronous machine and the infinite bus node, respectively; $\mathbf{y}_{10}, \mathbf{y}_{12}, \mathbf{y}_{20}$ are the three admittance elements, respectively, derived by using a $Y-\Delta$ transformation. 


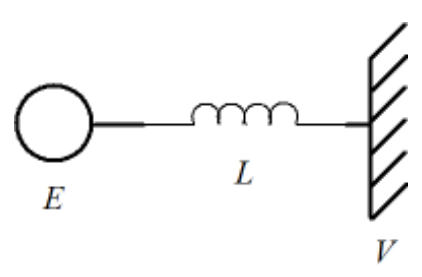

(a) A schematic representation of one machine connected to an infinite bus through a transmission line

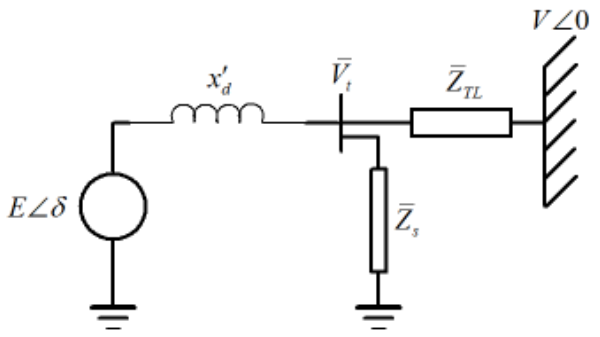

(b) The equivalent electrical circuit of one machine connected to an infinite bus through a transmission line

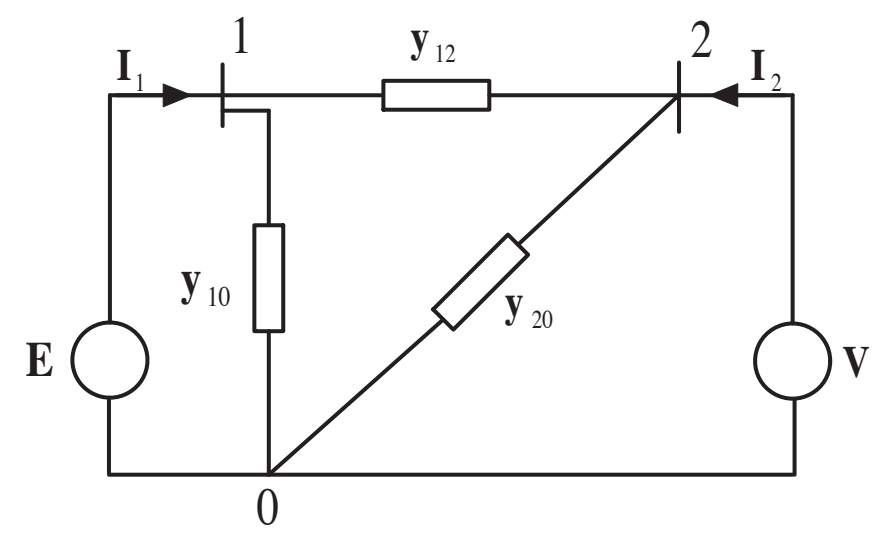

(c) Another equivalent circuit of one machine connected to an infinite bus through a transmission line

Figure A1. One machine connected to an infinite bus through a transmission line

The symbols with an arrow in the following equations are the vector variables corresponding to the Figure A. 1 (c). For node 1, according to Kirchhoff's law, we obtain:

$$
\mathbf{I}_{1}=(\mathbf{E}-\mathbf{V}) \mathbf{y}_{12}+\mathbf{E} \mathbf{y}_{10}=\mathbf{E} \mathbf{y}_{12}-\mathbf{V} \mathbf{y}_{12}+\mathbf{E} \mathbf{y}_{10}=\mathbf{E}\left(\mathbf{y}_{12}+\mathbf{y}_{10}\right)-\mathbf{V} \mathbf{y}_{12} \text {. }
$$

Define:

$$
\begin{aligned}
& \mathbf{Y}_{11}=Y_{11} \angle \theta_{11}=\mathbf{y}_{12}+\mathbf{y}_{10}, \theta_{11}=\arctan \left(\frac{\operatorname{real}\left(Y_{11}\right)}{\operatorname{imag}\left(Y_{11}\right)}\right) \\
& \mathbf{Y}_{12}=Y_{12} \angle \theta_{12}=-\mathbf{y}_{12}, \theta_{12}=\arctan \left(\frac{\operatorname{real}\left(Y_{12}\right)}{\operatorname{imag}\left(Y_{12}\right)}\right) .
\end{aligned}
$$

Then,

$$
\mathbf{I}_{1}=\mathbf{E} \mathbf{Y}_{11}+\mathbf{V} \mathbf{Y}_{12}=E Y_{11} e^{j\left(\delta+\theta_{11}\right)}+V Y_{12} e^{j \theta_{12}}
$$


So the power input $P_{e}$ at node 1 is

$$
\begin{gathered}
P_{e}=\operatorname{real}\left(\mathbf{E} \mathbf{I}_{1}^{*}\right) \\
\mathbf{E I}_{1}^{*}=E e^{j \delta}\left(E Y_{11} e^{-j\left(\delta+\theta_{11}\right)}+V Y_{12} e^{-j \theta_{12}}\right)=E^{2} Y_{11} e^{-j \theta_{11}}+E V Y_{12} e^{-j\left(\theta_{12}-\delta\right)} \\
P_{e}=\operatorname{real}\left(\mathbf{E I}_{1}^{*}\right)=E^{2} Y_{11} \cos \theta_{11}+E V Y_{12} \cos \left(\theta_{12}-\delta\right)
\end{gathered}
$$

When the voltage angle of the infinite bus is different from zero, we can also get the corresponding $P_{e}$, given as

$$
P_{e}=\operatorname{real}\left(\mathbf{E I}_{1}^{*}\right)=E^{2} Y_{11} \cos \theta_{11}+E V Y_{12} \cos \left(\theta_{12}+\alpha-\delta\right) .
$$

Now, we get the compact formula of the electrical output power of the generator as Eq. (A. 25). Define $\beta=\frac{\pi}{2}-\theta_{12}$ and $G_{11} \triangleq Y_{11} \cos \theta_{11}, G_{11}$ is the self-conductance, then Eq.(A. 25) is written as

$$
P_{e}=E^{2} G_{11}+E V Y_{12} \sin (\delta-\alpha+\beta) .
$$

Consequently, we can get the mathematical model of the generator node in the power system ${ }^{[14]}$ as

$$
\left(\frac{2 H}{\omega_{R}}\right) \dot{\omega}=P_{m}-P_{d}-P_{e}=P_{m}-D \omega-E^{2} G_{11}-E V Y_{12} \sin (\delta-\alpha+\beta) .
$$

In nodes power grid, the dynamic models of the generator nodes can be written as

$$
\left(\frac{2 H_{m i}}{\omega_{R}}\right) \dot{\omega}_{i}=P_{m i}-D_{i} \omega_{i}-\left(E_{i}^{2} G_{i i}+\sum_{j=1}^{n} E_{i} E_{j} Y_{i j} \sin \left(\delta_{i}-\delta_{j}+\varphi_{i j}\right)\right) .
$$

It can be also expressed as

$$
M_{i} \ddot{\theta}_{i}=P_{m i}-D_{i} \dot{\theta}_{i}-\left(E_{i}^{2} G_{i i}+\sum_{j=1}^{n} E_{i} E_{j} Y_{i j} \sin \left(\theta_{i}-\theta_{j}+\varphi_{i j}\right)\right),
$$

where $M_{i} \ddot{\theta}_{i}$ is the inertial term. When frequency synchronization analysis of (A. 29 ) is carried out, the inertia term $M_{i} \ddot{\theta}_{i}$ can be omitted due to the fact that it only affects the convergence time to synchronize the state but not its existence ${ }^{[14]}$. In addition, the resistive component is much smaller than the inductive component 
of the generators output impedance and the line impedance, then $\varphi_{i j}=0$. In consequence, the dynamic models of the generator nodes is given by

$$
D_{i} \dot{\theta}_{i}=P_{m i}-\left(E_{i}^{2} G_{i i}+\sum_{j=1}^{n} E_{i} E_{j} Y_{i j} \sin \left(\theta_{i}-\theta_{j}+\varphi_{i j}\right)\right),
$$

where, in terms of node $i, D_{i}$ and $M_{i}$ are the damping and inertia constants, respectively, $\theta_{i}$ of node $i$ is the angle of the shaft in radians with respect to a synchronously rotating reference, $\varphi_{i j}$ is the phase shifts caused by transfer conductance, $E_{i}$ is the voltage magnitude of the synchronous machine, $Y_{i j}$ is the admittance of the transmission line between nodes $i$ and $j$, and $G_{i i} \triangleq Y_{i i} \cos \theta_{i}$ is the self-conductance.

\section{Appendix B.}

The model for the droop controlled inverter connected RES node is the following.

Power grid typically contains a bank of RES nodes equipped with active powerfrequency droop inverter controllers operating in parallel. For the convenience of analysis, using two inverters in parallel as an example, Figure A.2 is the equivalent circuit for two inverters operating in parallel, where $E_{1}, E_{2}$ are the input voltage and $I_{1}, I_{2}$ are the input current of inverter 1 and 2, respectively. $R_{i}$ is the input impedance of inverter $i, j X_{i}$ is the line impedance between inverter and bus, $E$ is the voltage of load bus, $I_{0}, Z_{0}$ are the load current and load impedance, respectively, and $\delta_{1}, \delta_{2}$ are the voltage phase difference between inverter 1,2 and the bus, respectively. We get the inverter output active power from Figure B. 1.

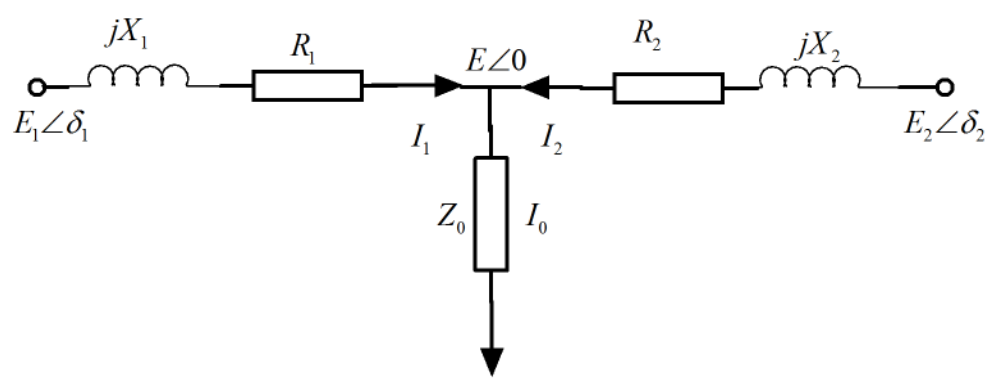

Figure B1. The equivalent circuit of two inverters operating in parallel

$$
P_{i}=\frac{R_{i} E_{i} E \cos \delta_{i}-R_{i} E^{2}}{X_{i}^{2}+R_{i}^{2}}+\frac{X_{i} E_{i} E}{X_{i}^{2}+R_{i}^{2}} \sin \delta_{i}, i=1,2 .
$$


Assume that the entire inverter system is inductive, the resistive portion of the inverter output impedance and line impedance is much smaller than the inductive portion, and assume that the two inverters output impedance are equal, i.e, $R_{1}=$ $R_{2} \approx 0$, then Eq. (B. 1) can be equivalently transformed as

$$
P_{i}=\frac{E_{i} E}{X_{i}} \sin \delta_{i}, i=1,2 .
$$

Compared with the load impedance, inverter source output reactance is very small, so the output voltage phase angle difference $\delta_{i}$ is also very small. Then we have $\sin \delta_{i} \approx \delta_{i}$, therefore Eq. (B. 2) can equivalently be given by :

$$
P_{i}=\frac{E_{i} E}{X_{i}} \delta_{i}, i=1,2 .
$$

Additionally, in a practical inverter system, the variation range of output voltage amplitude is not large, which can be approximately viewed as a constant, then the output power of inverter system is only related to the phase angle difference $\delta_{i}$

$$
\Delta P_{i}=\frac{E_{i} E}{X_{i}} \Delta \delta_{i}, i=1,2 .
$$

Since the phase control is achieved by adjusting the output frequency, the output frequency change of the inverter is $\Delta f_{i}=\frac{\Delta \omega_{i}}{2 \pi}=\frac{\Delta \delta_{i}}{2 \pi \Delta t}$, so we can change the inverter output phase by adjusting the output frequency, thereby, altering the active power output of the inverter.

Define

$$
n_{p_{i}}=\frac{X_{i}}{2 \pi E_{i} E},
$$

where the parameter $n_{p_{i}}$ is referred to as the droop coefficient, then the frequency droop control formula can be obtained

$$
f_{i}=f_{0_{i}}-n_{p_{i}} P_{i}
$$

where $f_{0_{i}}$ is the output frequency of the inverter without load.

\section{Appendix C.}


Table C.3: The nodes parameters of the North Local Power Grid of Shannxi Province

\begin{tabular}{|c|c|c|c|c|c|}
\hline \multirow{2}{*}{ Node Number } & \multirow{2}{*}{ Node type } & Node Voltage & Node phase & Node Power & Node Damping \\
\cline { 3 - 6 } & & $U(p . u)$. & $\delta\left(^{\circ}\right)$ & $P(p . u)$. & $D(p . u)$. \\
\hline 1 & 1 & 0.9259 & -1.3448 & 0.4950 & 3 \\
\hline 2 & 2 & 0.9492 & -11.7136 & -0.3658 & 2 \\
\hline 3 & 1 & 0.9679 & -13.8526 & 1.0000 & 3 \\
\hline 4 & 2 & 1.0012 & 3.1051 & -6.3509 & 2 \\
\hline 5 & 2 & 0.6971 & -2.4949 & -0.8509 & 2 \\
\hline 6 & 1 & 0.9543 & 5.0374 & 2.7000 & 3 \\
\hline 7 & 1 & 1.1242 & -8.9266 & 2.0000 & 3 \\
\hline 8 & 2 & 0.8933 & 19.0851 & -3.8909 & 2 \\
\hline 9 & 2 & 1.0934 & 1.2223 & -0.9609 & 2 \\
\hline 10 & 2 & 1.0350 & 10.4703 & -0.2609 & 2 \\
\hline 11 & 2 & 0.9971 & -2.2692 & -0.2209 & 2 \\
\hline 12 & 2 & 1.0182 & -1.6250 & -0.7609 & 2 \\
\hline 13 & 2 & 0.8435 & 6.9005 & -0.1809 & 2 \\
\hline 14 & 1 & 0.9915 & 5.5576 & 0.7500 & 3 \\
\hline 15 & 2 & 1.1604 & -11.2026 & -0.6309 & 2 \\
\hline 16 & 2 & 1.0098 & -15.3269 & -0.2009 & 2 \\
\hline 17 & 2 & 1.0041 & -10.9787 & -0.2409 & 2 \\
\hline 18 & 1 & 0.9266 & -14.1577 & 2.7000 & 3 \\
\hline 19 & 2 & 0.9969 & 0.5957 & -0.2109 & 2 \\
\hline 20 & 2 & 1.0232 & -4.1125 & -0.2259 & 2 \\
\hline 21 & 2 & 1.0426 & -3.6801 & -0.4809 & 2 \\
\hline 22 & 1 & 0.9627 & -13.6096 & 1.0000 & 3 \\
\hline & & & & & \\
\hline
\end{tabular}




\begin{tabular}{|c|c|c|c|c|c|}
\hline \multirow{2}{*}{ Node Number } & \multirow{2}{*}{ Node type } & Node Voltage & Node phase & Node Power & Node Damping \\
\hline & & $U($ p.u. $)$ & $\delta\left(^{\circ}\right)$ & $P(p . u)$. & $D($ p.u. $)$ \\
\hline 23 & 1 & 0.9764 & 7.7957 & 1.0000 & 3 \\
\hline 24 & 1 & 1.2024 & 4.3941 & 1.0000 & 3 \\
\hline 25 & 1 & 0.7742 & -0.8962 & 0.3000 & 3 \\
\hline 26 & 2 & 1.2229 & 10.2118 & -0.2409 & 2 \\
\hline 27 & 2 & 1.0338 & -8.7398 & -0.4009 & 2 \\
\hline 28 & 2 & 1.1000 & 4.1470 & -2.0609 & 2 \\
\hline 29 & 1 & 0.8336 & 3.4844 & 0.0200 & 3 \\
\hline 30 & 2 & 0.9410 & 3.4925 & -0.9609 & 2 \\
\hline 31 & 2 & 0.9722 & -7.2925 & -0.2609 & 2 \\
\hline 32 & 1 & 1.0423 & 3.2684 & 0.7200 & 3 \\
\hline 33 & 1 & 0.8330 & -5.1488 & 0.5000 & 3 \\
\hline 34 & 1 & 1.0472 & -8.9645 & 1.0000 & 3 \\
\hline 35 & 1 & 0.8787 & -12.0327 & 2.0000 & 3 \\
\hline 36 & 1 & 1.0066 & 10.3782 & 1.0000 & 3 \\
\hline 37 & 1 & 1.0652 & -8.4594 & 0.3000 & 3 \\
\hline 38 & 1 & 1.0327 & -1.7291 & 0.5000 & 3 \\
\hline 39 & 2 & 1.1083 & -12.0865 & -0.2009 & 2 \\
\hline 40 & 1 & 1.1006 & -2.9713 & 0.7500 & 3 \\
\hline 41 & 2 & 0.9349 & -32.3204 & -0.2609 & 2 \\
\hline 42 & 2 & 1.0257 & -10.8696 & -0.2009 & 2 \\
\hline 43 & 2 & 0.9056 & -14.2644 & -0.2609 & 2 \\
\hline 44 & 2 & 0.8678 & -10.1445 & -0.3209 & 2 \\
\hline 45 & 2 & 1.0925 & -2.1327 & -0.2809 & 2 \\
\hline 46 & 1 & 1.0000 & -3.2535 & 0.3000 & 3 \\
\hline 47 & 1 & 0.9945 & 19.4440 & 0.1200 & 3 \\
\hline 48 & 2 & 1.0911 & -5.7177 & -0.5609 & 2 \\
\hline 49 & 2 & 1.0595 & -2.5003 & -0.1809 & 2 \\
\hline 50 & 2 & 1.0350 & -15.6932 & -0.2009 & 2 \\
\hline 51 & 2 & 1.1250 & -4.7738 & -0.3109 & 2 \\
\hline 52 & 2 & 1.0930 & -13.3798 & -0.3609 & 2 \\
\hline 53 & 2 & 1.0240 & 0.3030 & -0.2609 & 2 \\
\hline 54 & 2 & 0.9310 & 8.5309 & -0.3409 & 2 \\
\hline 55 & 2 & 0.9348 & 4.0425 & -0.5609 & 2 \\
\hline 56 & 2 & 1.1192 & -7.0062 & -0.1809 & 2 \\
\hline 57 & 2 & 0.8388 & -16.3054 & -0.2109 & 2 \\
\hline 58 & 2 & 0.9976 & 14.6001 & -0.2209 & 2 \\
\hline 59 & 2 & 0.8051 & 20.5004 & -0.4609 & 2 \\
\hline 60 & 2 & 1.1020 & 1.2050 & -0.6609 & 2 \\
\hline
\end{tabular}




\begin{tabular}{|c|c|c|c|c|c|}
\hline \multirow{2}{*}{ Node Number } & \multirow{2}{*}{ Node type } & Node Voltage & Node phase & Node Power & Node Damping \\
\hline & & $U(p . u)$. & $\delta\left(^{\circ}\right)$ & $P(p . u)$. & $D(p . u)$. \\
\hline 61 & 1 & 1.0862 & -9.8990 & 0.7400 & 3 \\
\hline 62 & 1 & 1.0001 & 11.9777 & 0.7700 & 3 \\
\hline 63 & 1 & 0.9929 & -5.9266 & 0.4000 & 3 \\
\hline 64 & 2 & 0.7514 & -4.6981 & -0.5609 & 2 \\
\hline 65 & 2 & 1.0581 & 8.8638 & -0.2009 & 2 \\
\hline 66 & 1 & 0.7808 & -13.8522 & 1.0000 & 3 \\
\hline 67 & 2 & 0.7681 & -19.5675 & -0.2609 & 2 \\
\hline 68 & 2 & 1.0080 & 4.2068 & -0.6609 & 2 \\
\hline 69 & 2 & 0.9052 & 4.0074 & -0.4109 & 2 \\
\hline 70 & 2 & 1.0411 & 0.9514 & -0.2609 & 2 \\
\hline 71 & 1 & 1.0677 & 4.9668 & 0.8000 & 3 \\
\hline 72 & 1 & 1.0858 & 10.8224 & 0.3000 & 3 \\
\hline 73 & 2 & 0.9309 & 9.7045 & -0.3109 & 2 \\
\hline 74 & 2 & 1.0449 & -5.6857 & -0.2209 & 2 \\
\hline 75 & 2 & 1.0101 & 8.0997 & -0.2209 & 2 \\
\hline 76 & 1 & 1.0826 & 1.7325 & 0.6000 & 3 \\
\hline 77 & 1 & 1.0536 & -5.0554 & 0.2400 & 3 \\
\hline 78 & 1 & 1.0898 & -11.9331 & 0.5000 & 3 \\
\hline 79 & 1 & 0.9868 & 6.4697 & 0.4500 & 3 \\
\hline 80 & 1 & 0.9853 & -3.5362 & 0.3000 & 3 \\
\hline 81 & 2 & 1.1008 & 0.4643 & -0.4009 & 2 \\
\hline 82 & 2 & 0.7876 & -7.9295 & -0.4109 & 2 \\
\hline 83 & 2 & 0.9495 & -15.5051 & -0.3609 & 2 \\
\hline 84 & 1 & 0.8729 & 1.7159 & 0.5000 & 3 \\
\hline 85 & 1 & 0.9617 & -0.6214 & 0.5000 & 3 \\
\hline 86 & 1 & 1.0649 & 11.9903 & 0.2400 & 3 \\
\hline 87 & 1 & 1.0826 & 8.0170 & 0.5000 & 3 \\
\hline 88 & 1 & 0.8985 & 10.5330 & 0.5000 & 3 \\
\hline 89 & 1 & 0.9529 & -7.4888 & 0.1200 & 3 \\
\hline 90 & 1 & 1.0137 & -9.3633 & 0.6000 & 3 \\
\hline 91 & 1 & 0.9708 & -12.6909 & 1.9000 & 3 \\
\hline 92 & 1 & 1.0302 & 4.9798 & 0.4950 & 3 \\
\hline 93 & 1 & 1.0400 & 27.8908 & 0.4950 & 3 \\
\hline 94 & 1 & 0.9070 & 7.2757 & 1.2600 & 3 \\
\hline 95 & 1 & 0.9823 & -7.7306 & 0.0100 & 3 \\
\hline 96 & 1 & 0.7868 & 8.3663 & 0.9500 & 3 \\
\hline 97 & 1 & 1.1145 & -11.2833 & 0.4950 & 3 \\
\hline 98 & 2 & 0.9371 & -14.2447 & -2.0109 & 2 \\
\hline
\end{tabular}




\begin{tabular}{|c|c|c|c|c|c|}
\hline \multirow{2}{*}{ Node Number } & \multirow{2}{*}{ Node type } & Node Voltage & Node phase & Node Power & Node Damping \\
\cline { 3 - 6 } & & $U(p . u)$. & $\delta\left(^{\circ}\right)$ & $P(p . u)$. & $D(p . u)$. \\
\hline 99 & 1 & 0.8796 & 7.1744 & 0.6000 & 3 \\
\hline 100 & 1 & 0.9746 & -7.7791 & 0.9900 & 3 \\
\hline 101 & 1 & 0.8571 & 3.1599 & 0.4950 & 3 \\
\hline 102 & 1 & 0.9979 & 14.0654 & 0.8000 & 3 \\
\hline 103 & 1 & 0.9439 & 4.0112 & 1.0000 & 3 \\
\hline 104 & 1 & 1.2178 & 9.2966 & 0.9900 & 3 \\
\hline 105 & 2 & 1.1138 & -16.0580 & -1.9409 & 2 \\
\hline 106 & 2 & 0.7503 & 6.6154 & -1.1709 & 2 \\
\hline 107 & 1 & 1.0441 & 21.3850 & 0.9900 & 3 \\
\hline 108 & 1 & 0.8602 & 5.4114 & 0.9900 & 3 \\
\hline 109 & 2 & 0.9745 & -15.4088 & -2.6309 & 2 \\
\hline 110 & 1 & 1.0164 & -2.0314 & 1.5750 & 3 \\
\hline 111 & 1 & 1.0748 & -4.9997 & 0.9900 & 3 \\
\hline 112 & 2 & 0.9727 & 3.8302 & -0.9943 & 2 \\
\hline 113 & 2 & 1.1576 & 4.1204 & -6.9009 & 2 \\
\hline 114 & 1 & 0.9519 & 4.0549 & 0.4950 & 3 \\
\hline 115 & 1 & 1.0328 & -3.6378 & 0.9900 & 3 \\
\hline
\end{tabular}

Table C.4: The line parameters of the North Local Power Grid of Shannxi Province

\begin{tabular}{|c|c|c|}
\hline Source Node & Sink Node & Branch Reactance \\
\hline 1 & 104 & 0.0622 \\
\hline 1 & 109 & 0.0748 \\
\hline 1 & 111 & 0.0504 \\
\hline 2 & 112 & 0.0662 \\
\hline 3 & 112 & 0.0035 \\
\hline 4 & 5 & 0.0322 \\
\hline 4 & 7 & 0.0244 \\
\hline 4 & 59 & 0.0030 \\
\hline 5 & 7 & 0.0161 \\
\hline 5 & 105 & 0.0480 \\
\hline 6 & 113 & 0.0048 \\
\hline 8 & 44 & 0.3945 \\
\hline 8 & 53 & 0.1660 \\
\hline 8 & 74 & 1.0158 \\
\hline 8 & 83 & 0.1534 \\
\hline
\end{tabular}




\begin{tabular}{|c|c|c|}
\hline Source Node & Sink Node & Branch Reactance \\
\hline 8 & 87 & 0.1127 \\
\hline 9 & 14 & 0.0050 \\
\hline 9 & 18 & 0.0032 \\
\hline 9 & 20 & 0.0059 \\
\hline 9 & 52 & 0.0830 \\
\hline 9 & 59 & 0.0176 \\
\hline 9 & 61 & 0.0145 \\
\hline 9 & 62 & 0.0505 \\
\hline 9 & 82 & 0.0302 \\
\hline 10 & 11 & 0.6106 \\
\hline 10 & 27 & 1.0279 \\
\hline 10 & 30 & 0.1538 \\
\hline 10 & 46 & 0.2120 \\
\hline 10 & 68 & 0.1559 \\
\hline 11 & 27 & 0.2406 \\
\hline 11 & 30 & 0.0803 \\
\hline 11 & 46 & 0.0496 \\
\hline 11 & 72 & 0.0099 \\
\hline 11 & 79 & 0.0154 \\
\hline 12 & 17 & 0.0449 \\
\hline 12 & 39 & 0.0160 \\
\hline 13 & 74 & 0.1819 \\
\hline 13 & 86 & 0.0314 \\
\hline 13 & 109 & 0.0526 \\
\hline 15 & 32 & 0.0409 \\
\hline 15 & 65 & 0.6658 \\
\hline 15 & 66 & 0.0517 \\
\hline 15 & 69 & 0.1042 \\
\hline 15 & 88 & 0.0040 \\
\hline 16 & 41 & 0.0295 \\
\hline 16 & 52 & 0.1940 \\
\hline 16 & 67 & 0.0483 \\
\hline 17 & 39 & 0.0656 \\
\hline 17 & 40 & 0.0431 \\
\hline 17 & 47 & 0.0115 \\
\hline 17 & 57 & 0.0431 \\
\hline 18 & 65 & 0.0865 \\
\hline 18 & 69 & 0.1681 \\
\hline
\end{tabular}




\begin{tabular}{|c|c|c|}
\hline Source Node & Sink Node & Branch Reactance \\
\hline 19 & 31 & 0.2210 \\
\hline 19 & 86 & 0.0539 \\
\hline 21 & 34 & 0.0014 \\
\hline 21 & 48 & 0.0197 \\
\hline 21 & 68 & 0.0208 \\
\hline 21 & 113 & 0.0156 \\
\hline 22 & 58 & 0.0177 \\
\hline 23 & 67 & 0.0078 \\
\hline 24 & 65 & 0.0064 \\
\hline 25 & 50 & 0.0023 \\
\hline 26 & 44 & 0.0647 \\
\hline 27 & 30 & 0.6714 \\
\hline 27 & 46 & 0.0835 \\
\hline 27 & 68 & 0.0630 \\
\hline 28 & 30 & 0.0088 \\
\hline 28 & 76 & 0.0083 \\
\hline 28 & 85 & 0.0071 \\
\hline 29 & 30 & 0.0500 \\
\hline 30 & 46 & 0.1385 \\
\hline 30 & 50 & 0.0428 \\
\hline 30 & 59 & 0.1369 \\
\hline 30 & 67 & 0.0293 \\
\hline 31 & 75 & 0.0061 \\
\hline 31 & 81 & 0.0317 \\
\hline 31 & 86 & 2.2736 \\
\hline 31 & 109 & 0.0263 \\
\hline 32 & 55 & 0.0638 \\
\hline 33 & 41 & 0.0005 \\
\hline 35 & 50 & 0.0004 \\
\hline 36 & 69 & 0.0107 \\
\hline 37 & 65 & 0.0008 \\
\hline 38 & 42 & 0.0010 \\
\hline 39 & 54 & 0.0171 \\
\hline 39 & 80 & 0.0271 \\
\hline 40 & 57 & 0.0442 \\
\hline 40 & 60 & 0.0105 \\
\hline 40 & 62 & 0.0182 \\
\hline 41 & 48 & 0.0650 \\
\hline
\end{tabular}




\begin{tabular}{|c|c|c|}
\hline Source Node & Sink Node & Branch Reactance \\
\hline 42 & 51 & 0.0257 \\
\hline 42 & 78 & 0.0071 \\
\hline 43 & 51 & 0.0050 \\
\hline 43 & 74 & 0.0855 \\
\hline 44 & 74 & 0.0768 \\
\hline 44 & 83 & 2.0159 \\
\hline 44 & 87 & 0.1698 \\
\hline 45 & 49 & 0.0200 \\
\hline 45 & 54 & 0.0399 \\
\hline 48 & 64 & 0.0058 \\
\hline 48 & 74 & 0.1036 \\
\hline 48 & 113 & 0.0364 \\
\hline 50 & 82 & 0.0293 \\
\hline 51 & 68 & 0.0201 \\
\hline 51 & 71 & 0.0053 \\
\hline 51 & 74 & 0.0796 \\
\hline 52 & 59 & 0.0374 \\
\hline 52 & 61 & 0.0696 \\
\hline 52 & 67 & 0.0483 \\
\hline 52 & 73 & 0.0706 \\
\hline 52 & 77 & 0.0055 \\
\hline 53 & 70 & 0.0568 \\
\hline 54 & 69 & 0.0371 \\
\hline 55 & 73 & 0.0569 \\
\hline 56 & 58 & 0.1162 \\
\hline 56 & 81 & 0.1376 \\
\hline 56 & 109 & 0.0979 \\
\hline 58 & 68 & 0.0936 \\
\hline 59 & 67 & 0.0516 \\
\hline 60 & 62 & 0.0293 \\
\hline 60 & 89 & 0.0061 \\
\hline 62 & 82 & 0.0290 \\
\hline 62 & 84 & 0.0004 \\
\hline 63 & 107 & 0.0384 \\
\hline 63 & 109 & 0.0022 \\
\hline 65 & 66 & 0.0713 \\
\hline 65 & 69 & 0.7989 \\
\hline
\end{tabular}




\begin{tabular}{|c|c|c|}
\hline Source Node & Sink Node & Branch Reactance \\
\hline 66 & 69 & 0.0621 \\
\hline 8 & 113 & 0.0428 \\
\hline 74 & 83 & 5.1912 \\
\hline 74 & 87 & 0.4373 \\
\hline 81 & 109 & 0.0263 \\
\hline 83 & 87 & 0.5760 \\
\hline 90 & 105 & 0.0196 \\
\hline 91 & 102 & 0.0044 \\
\hline 92 & 97 & 0.0237 \\
\hline 93 & 96 & 0.0089 \\
\hline 94 & 96 & 0.0141 \\
\hline 95 & 96 & 0.0283 \\
\hline 96 & 101 & 0.0089 \\
\hline 96 & 115 & 0.0089 \\
\hline 97 & 98 & 0.0500 \\
\hline 97 & 100 & 0.0889 \\
\hline 98 & 99 & 0.0433 \\
\hline 100 & 109 & 0.0320 \\
\hline 100 & 114 & 0.0592 \\
\hline 102 & 103 & 0.0500 \\
\hline 103 & 106 & 0.0065 \\
\hline 105 & 106 & 0.0500 \\
\hline 108 & 109 & 0.1189 \\
\hline 109 & 110 & 0.0141 \\
\hline 112 & 113 & 0.0500 \\
\hline 114 & 115 & 0.0500 \\
\hline & & \\
\hline
\end{tabular}

